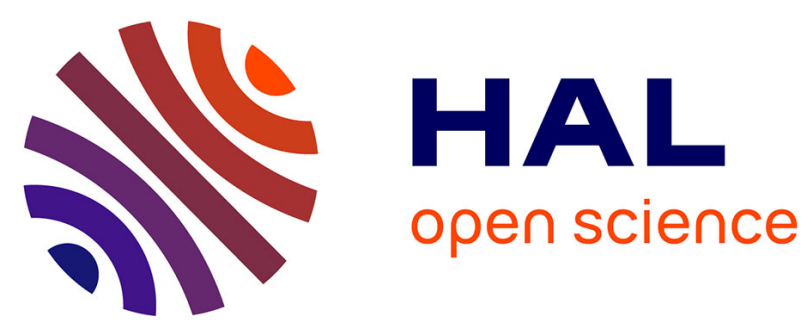

\title{
Polarimetric target detection in the presence of spatially fluctuating Mueller matrices
}

Guillaume Anna, François Goudail, Daniel Dolfi

\section{To cite this version:}

Guillaume Anna, François Goudail, Daniel Dolfi. Polarimetric target detection in the presence of spatially fluctuating Mueller matrices. Optics Letters, 2011, 36 (23), pp.4590-4592. hal-00747158

\section{HAL Id: hal-00747158 \\ https://hal-iogs.archives-ouvertes.fr/hal-00747158}

Submitted on 30 Oct 2012

HAL is a multi-disciplinary open access archive for the deposit and dissemination of scientific research documents, whether they are published or not. The documents may come from teaching and research institutions in France or abroad, or from public or private research centers.
L'archive ouverte pluridisciplinaire HAL, est destinée au dépôt et à la diffusion de documents scientifiques de niveau recherche, publiés ou non, émanant des établissements d'enseignement et de recherche français ou étrangers, des laboratoires publics ou privés. 


\title{
Polarimetric target detection in the presence of spatially fluctuating Mueller matrices
}

\author{
Guillaume Anna, ${ }^{1}$ François Goudail, ${ }^{1, *}$ and Daniel Dolfi ${ }^{2}$ \\ ${ }^{1}$ Laboratoire Charles Fabry, UMR 8501, Institut d'Optique, CNRS, Université Paris Sud 11, 91127 Palaiseau, France \\ ${ }^{2}$ Thales Research and Technology-France, RD128, 91767 Palaiseau Cedex, France \\ *Corresponding author: francois.goudail@institutoptique.fr
}

Received September 9, 2011; revised October 20, 2011; accepted October 23, 2011; posted October 24, 2011 (Doc. ID 154458); published November 28, 2011

\begin{abstract}
In polarimetric imaging systems, the main source of perturbations may not be detection noise but fluctuations of the Mueller matrices in the scene. In this case, we propose a method for determining the illumination and analysis polarization states that allow reaching the highest target detection performance. We show with simulations and real-world images that, in practical applications, the statistics of Mueller matrix fluctuations have to be taken into account to optimize polarimetric imagery. (c) 2011 Optical Society of America

OCIS codes: $110.5405,100.0100$.
\end{abstract}

Polarimetric images are useful for gathering information that is not visible on intensity images and can be useful in such domains as machine vision, remote sensing, biomedical imaging, and industrial control [1,2]. In many applications, the objective is to discriminate a target from its background, and a lot of efforts have been done to perform this task in an optimal way. The first results have been obtained in the radar community, where images are mainly perturbed by multiplicative speckle noise $[3,4]$. More recently, this problem has been addressed in the optics community, and the cases of additive detector noise [5] and of Poisson shot noise [6] have been investigated. However, in many situations such as target detection in foliage or in biological tissues, the dominant source of perturbations is not due to detector, speckle, or shot noise but to the fluctuations of the Mueller matrices in the scene. For the first time to our knowledge in the optical domain, we address the theoretical aspects of this issue and demonstrate on simulated and real-world images that, in practical applications, the statistics of spatial Mueller matrix fluctuations have to be taken into account to optimize polarimetric imagery.

We consider an active polarimetric imaging system that illuminates the scene with light whose polarization state is defined by a Stokes vector $\mathbf{S}$ and is produced by a polarization state generator (PSG) (see Fig. 1). The polarimetric properties of a region of the scene corresponding to a pixel in the image is characterized by its Mueller matrix $M$. The Stokes vector of the light scattered by this region is $\mathbf{S}^{\prime}=M \mathbf{S}$. It is analyzed by a polarization state analyzer (PSA), which is a generalized polarizer whose eigenstate is the Stokes vector $\mathbf{T}$. The number of photoelectrons measured at a pixel of the sensor is

$$
i=\frac{\eta I_{0}}{2} \mathbf{T}^{T} M \mathbf{S},
$$

where the superscript $T$ denotes matrix transposition. In this equation, $\mathbf{S}$ and $\mathbf{T}$ are unit intensity, purely polarized Stokes vectors, $I_{0}$ is a number of photons, and $\eta$ is the conversion efficiency between photons and electrons.

We will consider that the image is disturbed by two different types of noise. The first one is additive sensor noise, which will be assumed of zero mean and variance $\sigma^{2}$. The second one is due to the fact that, from one pixel to the next, the Mueller matrix randomly fluctuates around its average value. This spatial fluctuation can lead to a significant noise disturbing the intensity image and thus has to be taken into account. For the sake of simplicity, we will assume that the scene is composed of two regions: a target characterized by an average Mueller matrix $\left\langle M^{a}\right\rangle$ and a background characterized by an average Mueller matrix $\left\langle M^{b}\right\rangle$. Each pixel belonging to region $u \in$ $\{a, b\}$ has a Mueller matrix $M$ that deviates from the average matrix $\left\langle M^{u}\right\rangle$ of this region. These spatial fluctuations are characterized by their correlation matrices defined as

$$
G_{u}=\left\langle\left(\mathbf{V}_{M}-\mathbf{V}_{\left\langle M^{u}\right\rangle}\right)\left(\mathbf{V}_{M}-\mathbf{V}_{\left\langle M^{u}\right\rangle}\right)^{T}\right\rangle,
$$

with $\mathbf{V}_{M}$ the 16-component vector obtained by reading the Mueller matrix $M$ in the lexicographic order. Using this notation and taking into account additive noise, Eq. (1) can be written as

$$
i=\frac{\eta I_{0}}{2}[\mathbf{T} \otimes \mathbf{S}]^{T} \mathbf{V}_{M}+n,
$$

where $\otimes$ denotes the Kronecker product and $n$ is a random variable of zero mean and variance $\sigma^{2}$. Notice that $i$ is now a random variable whose statistical properties depend on the region where the pixel is located. If we assume that the fluctuations of $\mathbf{V}_{M}$ and $n$ are independent, the mean and variance of $i$ in region $u \in\{a, b\}$ are given by

$$
\langle i\rangle_{u}=\eta I_{0} / 2 \times[\mathbf{T} \otimes \mathbf{S}]^{T} \mathbf{V}_{\left\langle M^{u}\right\rangle},
$$

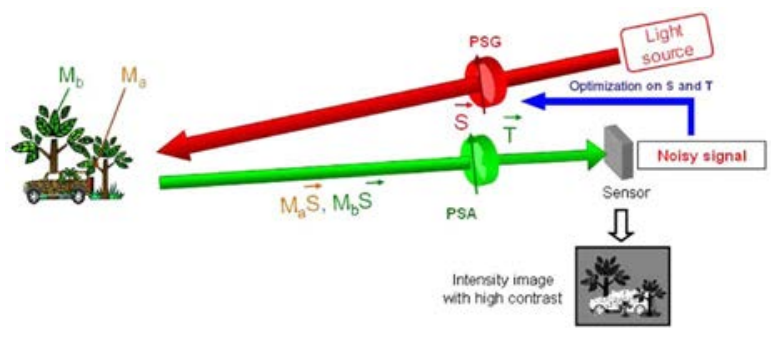

Fig. 1. (Color online) Polarimetric imaging setup. 


$$
\operatorname{var}[i]_{u}=\left(\eta I_{0} / 2\right)^{2} \times[\mathbf{T} \otimes \mathbf{S}]^{T} G_{u}[\mathbf{T} \otimes \mathbf{S}]+\sigma^{2} .
$$

Our objective is to optimize the discrimination between the target and the background region in the intensity image. To quantify the quality of this discrimination, we will use the Fisher ratio [7]. Using the notation defined above, it is defined as

$$
\mathcal{F}(\mathbf{S}, \mathbf{T})=\frac{\left[\langle i\rangle_{a}-\langle i\rangle_{b}\right]^{2}}{\operatorname{var}[i]_{a}+\operatorname{var}[i]_{b}} .
$$

By using Eqs. (3)-(), it can be put in the following form:

$$
\mathcal{F}(\mathbf{S}, \mathbf{T})=\frac{[\mathbf{T} \otimes \mathbf{S}]^{T} G_{\text {target }}[\mathbf{T} \otimes \mathbf{S}]}{[\mathbf{T} \otimes \mathbf{S}]^{T} G_{\text {fluct }}[\mathbf{T} \otimes \mathbf{S}]+8 / \mathrm{SNR}},
$$

where $G_{\text {fluct }}=G_{a}+G_{b}$ is the average covariance matrix of the Mueller matrix fluctuations over the scene, $G_{\text {target }}=\left(\left\langle M^{a}\right\rangle-\left\langle M^{b}\right\rangle\right)\left(\left\langle M^{a}\right\rangle-\left\langle M^{b}\right\rangle\right)^{T}$ is the interclass covariance matrix, which represents the difference between the average Mueller matrices of the two regions, and $\operatorname{SNR}=\left(\eta^{2} I_{0}^{2}\right) / \sigma^{2}$ is the signal-to-noise ratio due to the presence of additive noise.

Our goal will be to determine the optimal couple of illumination and analysis states $(\mathbf{S}, \mathbf{T})$ that maximizes the function $\mathcal{F}(\mathbf{S}, \mathbf{T})$ defined in Eq. (7). In order to show the importance of taking into account the actual statistics of Mueller matrix fluctuations in this optimization, we will take one example based on simulation and another one based on real-world polarimetric images.

In order to simulate random Mueller matrices, let us use the Lu-Chipman decomposition [8], which consists in describing each Mueller matrix as a product of three components: retarder $\left(M_{R}\right)$, diattenuator $\left(M_{D}\right)$, and depolarizer $\left(M_{\Delta}\right)$. The resulting Mueller matrix $M$ is then given by $M=M_{\Delta} M_{R} M_{D}$. We will assume that the Mueller matrices of the diattenuator and the retarder are constant in each region and only the diagonal coefficients of the depolarizer matrices $M_{\Delta}^{u}$ randomly fluctuate. The standard deviations of these fluctuations in the two regions are defined in Table 1 . We generate two sets of $N=5000$ Mueller matrices according to this model. The average Mueller matrices of regions $a$ and $b$ are estimated as

$$
\begin{aligned}
& \left\langle M_{a}\right\rangle \quad\left\langle M_{b}\right\rangle \\
& {\left[\begin{array}{cccc}
0.64 & 0.00 & 0.01 & 0.00 \\
0.00 & 0.31 & 0.03 & 0.03 \\
0.00 & -0.03 & 0.24 & 0.02 \\
0.00 & -0.01 & -0.02 & 0.16
\end{array}\right]\left[\begin{array}{cccc}
0.60 & 0.01 & 0.00 & 0.00 \\
0.00 & 0.19 & 0.00 & -0.02 \\
0.00 & 0.00 & 0.15 & 0.00 \\
0.00 & 0.02 & 0.00 & 0.22
\end{array}\right]}
\end{aligned}
$$

They are mainly depolarizing but with depolarization coefficients that are anisotropic and region dependent.

Table 1. Standard Deviation of the Fluctuations of the Diagonal Coefficients of the Depolarizer Matrices $M_{\Delta}^{u}$

\begin{tabular}{ccccc}
\hline Region & $M_{\Delta, 00}$ & $M_{\Delta, 11}$ & $M_{\Delta, 22}$ & $M_{\Delta, 33}$ \\
\hline $\mathrm{a}$ & 0.05 & 0.1 & 0.05 & 0.01 \\
$\mathrm{~b}$ & 0.05 & 0.03 & 0.02 & 0.02 \\
\hline
\end{tabular}

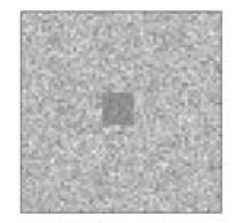

(a) $\mathcal{F}=2.3$

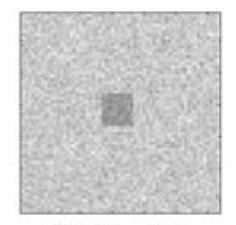

(b) $\mathcal{F}=4.1$
Fig. 2. (a) Optimal image taking into account only additive noise. (b) Optimal image taking into account Mueller matrix fluctuations.

Let us first assume that the fluctuations of Mueller matrices are neglected in the optimization process; that is, $G_{\text {fluct }}$ is assumed to be zero. The SNR of additive noise defined in Eq. (7) is assumed equal to $\left(\eta I_{0} / \sigma\right)^{2}=15^{2}$. The optimal states maximizing the Fisher ratio are presented in the first row of Table 2 ( $\alpha$ denotes the azimuth of a polarization state and $\varepsilon$ its ellipticity). The value of the Fisher ratio obtained with this couple of optimal PSG and PSA states is $\mathcal{F}=2.3$, and the image obtained appears in Fig. 2(a). Let us now take into account the fact that the Mueller matrices fluctuate inside each region. The covariance matrices $G_{a}$ and $G_{b}$ of these fluctuations are estimated from the generated data sets, and these estimates are used in Eq. (7). The obtained optimal states are presented in the second row of Table 2: it is seen that they are different from those obtained without taking into account the Mueller matrix fluctuations. They lead to a better value of the Fisher ratio $\mathcal{F}=4.1$, as can be seen on the scalar image obtained using this couple of optimal states in Fig. 2(b). In order to interpret this result, let us look at the matrices in Eq. (8). The difference between the two average Mueller matrices is higher for the coefficient $M_{11}$, and thus the optimal states maximizing the contrast when taking into account only the additive noise are roughly linear with azimuth $90^{\circ}$. However, we can notice in Table 1 that it is also for coefficient $M_{11}$ that the Mueller matrix fluctuations are the highest. Thus, when these fluctuations are taken into account, the optimal states correspond to the best compromise between maximization of the separability of the average intensities and minimization of the fluctuations. It is seen in Table 2 that these states are elliptic with nonparallel azimuth.

Let us now consider a real-world polarimetric imaging scenario. The observed scene is a piece of translucent birefringent plastic with spatial fluctuations, on the backside of which two pieces of translucent adhesive tape have been stuck on a polarizer. The target regions are constituted by the pieces of adhesive [see Fig. 3(a)]. The standard intensity image of this scene is shown in Fig. 3(b): the adhesive tapes are not visible on it. The scene is now observed with a polarimetric imager of the type represented in Fig. 1 . The average and covariance matrices of the Mueller matrices of the region $a$ (target)

Table 2. Optimal States Obtained by Taking into Account or Not the Fluctuations of the Polarimetric Properties of the Scene

\begin{tabular}{lllll}
\hline Algorithm & $\alpha_{S}$ & $\varepsilon_{S}$ & $\alpha_{T}$ & $\varepsilon_{T}$ \\
\hline$G_{\text {fluct }}=0$ & $-90^{\circ}$ & $-10^{\circ}$ & $85^{\circ}$ & $10^{\circ}$ \\
$G_{\text {fluct }} \neq 0$ & $-30^{\circ}$ & $-21^{\circ}$ & $20^{\circ}$ & $-19^{\circ}$ \\
\hline
\end{tabular}




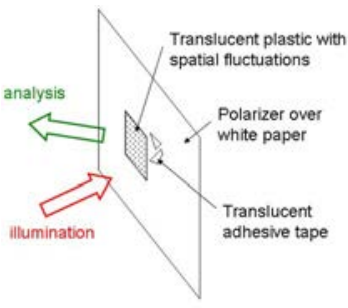

(a)

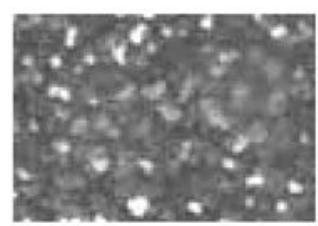

(b)
Fig. 3. (Color online) (a) Scheme of the observed scene. (b) Intensity image of the scene.

and the region $b$ (background) are estimated thanks to a database collected previously and containing sets of Mueller matrices associated with the two regions that have to be discriminated. These Mueller matrices are acquired by generating 16 different configurations of the PSG and PSA states [9].

Based on these estimations, we compute the PSA and PSG states that maximize the contrast under two noise hypotheses: additive noise only, which leads to $\left(\alpha_{S}, \epsilon_{S}\right)=$ $\left(15^{\circ},-30^{\circ}\right)$ and $\left(\alpha_{T}, \epsilon_{T}\right)=\left(-90^{\circ},-35^{\circ}\right)$, and Mueller matrix fluctuations, which leads to $\left(\alpha_{S}, \epsilon_{S}\right)=\left(-35^{\circ},-15^{\circ}\right)$ and $\left(\alpha_{T}, \epsilon_{T}\right)=\left(-50^{\circ},-10^{\circ}\right)$. It is noticeable that these two pairs of states are different. By implementing them on the polarimetric imager, we obtain the scalar polarimetric images represented in Fig. 4 . It is seen that the optimal states taking into account only additive noise reveals adhesive pieces by maximizing the difference between of average intensity coming from the target and the background $(\Delta=1100)$. However, the presence of high fluctuations decreases the contrast. On the other hand, using the optimal states that take into account the specific statistics of Mueller matrix fluctuations, the difference between the average intensity is only about $(\Delta=500)$, but, as the fluctuations are much lower, the contrast is better. This is a clear illustration that correctly optimized polarimetric imagers can help to enhance the contrast in the presence of spatial fluctuations.

Let us now briefly address the computational issues for estimation of the optimal states. Since there is no closedform solution to optimization of Eq. (7), one has to use a numerical algorithm. According to our experience, the function $\mathcal{F}(\mathbf{S}, \mathbf{T})$ may have more than one local maximum but always has smooth variations. We have thus designed an iterative code with a progressive mesh. For a given value of the analysis state $\mathbf{T}$ (the same reasoning can be done by fixing the illumination state), the illumination state $\mathbf{S}$ maximizing the contrast can be found as follows. First, the whole Poincaré sphere is swept using a sampling of $10^{\circ}$ for the azimuth and the ellipticity of the polarization state. The two highest local maxima that are

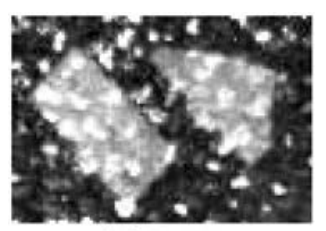

(a) $\mathcal{F}=1.2$

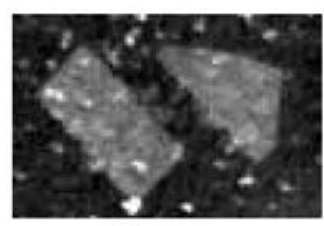

(b) $\mathcal{F}=8.2$
Fig. 4. (a) Optimal image of the scene in Fig. 3 taking into account only additive noise. (b) Optimal image taking into account Mueller matrix fluctuations.

separated of minimum $20^{\circ}$ are kept. Then around each of these two maxima, a $20^{\circ} \times 20^{\circ}$ wide area in azimuth and ellipticity is swept with a sampling step of $5^{\circ}$, and the two states leading to the highest contrast are kept. Finally, around these two states, a $10^{\circ} \times 10^{\circ}$ wide area is swept with a sampling step of $1^{\circ}$, and the state leading to the highest contrast is kept. This sequence is alternatively performed on $\mathbf{S}$ and $\mathbf{T}$ in order to obtain a result in about $2 \mathrm{~s}$ with a precision of $1^{\circ}$, which is sufficiently close to the globally optimal performance.

In conclusion, the fluctuations of the polarimetric properties of the scene induce a noise that can be more detrimental to polarimetric target detection than additive sensor noise. We have proposed a methodology that addresses successfully this issue and makes it possible to optimize polarimetric imaging systems in the presence of such heavy fluctuations. It opens up perspectives for optimal adaptation of polarimetric imaging systems to difficult applications such has imaging through diffusing media or decamouflaging. Interesting perspectives include generalization to the discrimination of more than two regions.

G. Anna's Ph.D. dissertation is supported by the Délégation Générale pour l'Armement (DGA), Mission pour la Recherche et l'Innovation Scientifique (MRIS).

\section{References}

1. J. S. Tyo, M. P. Rowe, E. N. Pugh, and N. Engheta, Appl. Opt. 35, 1855 (1996).

2. J. E. Solomon, Appl. Opt. 20, 1537 (1981).

3. A. A. Swartz, H. A. Yueh, J. A. Kong, L. M. Novak, and R. T. Shin, J. Geophys. Res. 93, 15252 (1988).

4. J. Yang, Y. Yamaguchi, W. Boerner, and S. Lin, IEEE Trans. Geosci. Remote Sens. 38, 965 (2000).

5. F. Goudail and A. Bénière, Opt. Lett. 34, 1471 (2009).

6. F. Goudail, Opt. Lett. 35, 2600 (2010).

7. K. Fukunaga, Introduction to Statistical Pattern Recognition (Academic, 1990).

8. S. Y. Lu and R. A. Chipman, J. Opt. Soc. Am. A 13, 1106 (1996).

9. D. Goldstein, Polarized Light (Marcel Dekker, 2003). 